9-1-2016

\title{
Seizure clusters in drug-resistant focal epilepsy.
}

\author{
Ali Akbar Asadi-Pooya \\ Thomas Jefferson University \\ maromi nei \\ Jefferson Medical College \\ Ashwini Sharan \\ Thomas Jefferson University \\ Michael R. Sperling \\ Thomas Jefferson University
}

Follow this and additional works at: https://jdc.jefferson.edu/neurologyfp

Part of the Neurology Commons

Let us know how access to this document benefits you

\section{Recommended Citation}

Asadi-Pooya, Ali Akbar; nei, maromi; Sharan, Ashwini; and Sperling, Michael R., "Seizure clusters in drug-resistant focal epilepsy." (2016). Department of Neurology Faculty Papers. Paper 122.

https://jdc.jefferson.edu/neurologyfp/122

This Article is brought to you for free and open access by the Jefferson Digital Commons. The Jefferson Digital Commons is a service of Thomas Jefferson University's Center for Teaching and Learning (CTL). The Commons is a showcase for Jefferson books and journals, peer-reviewed scholarly publications, unique historical collections from the University archives, and teaching tools. The Jefferson Digital Commons allows researchers and interested readers anywhere in the world to learn about and keep up to date with Jefferson scholarship. This article has been accepted for inclusion in Department of Neurology Faculty Papers by an authorized administrator of the Jefferson Digital Commons. For more information, please contact: JeffersonDigitalCommons@jefferson.edu. 


\section{Brief Communications}

Title: Seizure clusters in drug-resistant focal epilepsy

Ali A. Asadi-Pooya, M.D., Maromi Nei , M.D. Ashwini Sharan, M.D., Michael R. Sperling,

M.D.

Jefferson Comprehensive Epilepsy Center, Department of Neurology, Thomas Jefferson

University, Philadelphia, Pennsylvania, U.S.A.

\section{Address for correspondence:}

Ali A. Asadi-Pooya, M.D.

Department of Neurology

901 Walnut Street, Suite 435

Philadelphia, PA 19107

Phone: 215-955-1222

Fax: 215-955-3745

E-mail: aliasadipooya@yahoo.com; maromi.nei@jefferson.edu; Ashwini.Sharan@jefferson.edu; michael.sperling@jefferson.edu

Running title: Seizure clusters

Key words: Cluster; Epilepsy; Outcome; Seizure; Surgery

Number of text pages: 6; Word count for the paper: 1725. Word count for the abstract: 195.

Character count for the title: 49; Number of references: 13; Number of tables: 1; Number of figures: 1 


\begin{abstract}
:
We investigated clinical factors associated with seizure clustering in patients with drug-resistant focal epilepsy and any association between seizure clustering and outcome after surgery. We performed a retrospective study including patients with a diagnosis of drug-resistant focal epilepsy who underwent epilepsy surgery. Patients were prospectively registered in a database from 1986 until 2015. Seizure cluster was defined as two or more seizures occurring within two days. Potential risk factors for seizure clustering were assessed. To investigate any potential association between seizure clusters and seizure outcome after surgery, time to event analysis was used to produce a Kaplan-Meier estimate of seizure recurrence. We studied 764 patients. Seizure clusters were reported in $23.6 \%$ of TLE patients and $16.9 \%$ of extratemporal patients (p $=0.2$. We could not identify any significant clinical factors associated with seizure clustering. Among patients with TLE, those who had history of seizure clusters fared better after surgery (p $<0.01)$. We found that seizure clusters relate to prognosis after anterior temporal lobectomy in drug-resistant temporal lobe epilepsy. This data may provide added value for surgical prognostication when combined with other data types. A better understanding of the neurobiology underlying seizure clusters is needed.
\end{abstract}

Key words: Cluster; Epilepsy; Outcome; Seizure; Surgery 


\section{Introduction}

Seizure cluster is defined as a closely grouped series of seizures ${ }^{1}$. There is no universally accepted definition for a cluster of seizures and published studies have used varying definitions, including two to four seizures per $<48$ hours; 3 seizures per 24 hours; or having several convulsions within a day or two ${ }^{1}$. Seizure clustering in patients with epilepsy is a relatively common phenomenon with a reported prevalence ranging from $20 \%$ to $30 \%$ in some previous studies ${ }^{2,3}$. Seizure clusters may impose significant risks to patients with epilepsy. They were associated with status epilepticus, hospitalizations, and worse seizure control ${ }^{3}$. Whether a pattern of seizure clustering influences seizure outcome after surgery for drug-resistant seizures in patients with focal epilepsy is not known.

It is important to investigate the clinical features associated with seizure clusters as this may help elucidate the underlying pathophysiology. Similarly, it is desirable to know whether seizure clusters relate to seizure outcome after surgery for drug-resistant seizures. We address these two issues in the present investigation.

\section{Methods}

We performed a retrospective study including patients with a diagnosis of drug-resistant focal epilepsy who underwent epilepsy surgery at Jefferson Comprehensive Epilepsy Center. Adult and adolescent patients (above 10 years of age) were prospectively registered in a database from 1986 until 2015. The diagnosis of epilepsy was made by the epileptologists working at this institution. Individuals with progressive neurological disease, malignant brain tumors, or psychogenic nonepileptic seizures were excluded. Patients had a comprehensive presurgical 
evaluation including a brain MRI, scalp video-EEG monitoring, neuropsychological testing, and other appropriate testing as clinically indicated ${ }^{4}$. All patients had resective brain surgery. Patients were followed for up to five years after surgery (in many patients data were missing beyond five years of follow-up for a valid analysis). Seizure cluster was defined as two or more seizures occurring within two days, in a habitual pattern (i.e., not only in response to medication withdrawal or concurrent illness) that was also distinguishable from more sporadic usual seizures. It was derived from the written histories obtained by physicians and encoded in the database as "Yes/No". Seizure outcome was obtained during office visits, telephone contact, and by letter. Postsurgical outcome was classified into two groups: no recurrence of complex partial (CPS) or secondarily generalized seizures (though auras could recur) or relapse of CPS or secondarily generalized seizures at any time after surgery. Age, gender, race, epilepsy risk factors [e.g., history of febrile seizures in childhood, any family history of epilepsy, etc.], age at seizure onset, seizure type(s) and history, date of surgery, date of the first relapse (if any) and date of the last contact with all patients were registered routinely.

Potential risk factors for seizure clustering (i.e., gender, age at surgery, duration of epilepsy, history of tonic-clonic seizures before surgery, history of febrile seizures in childhood, family history of epilepsy, full scale intelligence quotient (IQ), preoperative auras, and MRI findings) were assessed comparing patients who had clusters and those who did not have clusters. We performed Pearson Chi-square, Mann-Whitney, Kolmogorov-Smirnov, and t-test depending upon the circumstance. To investigate any potential association between seizure clusters and outcome after surgery, time to event analysis was used to produce a Kaplan-Meier estimate of seizure recurrence. The Cox-Mantel test was used to compare the cumulative time-dependent probability of occurrence of the first seizure after resective epilepsy surgery in patients with and 
without clusters. In a subanalysis, we investigated the rate of seizure freedom in the last year of follow-up in patients with at least two years of postoperative follow-up. We classified the patients as seizure free if they had no CPS or secondarily generalized seizures for the last year of follow-up. $\mathrm{P}$ values less than 0.05 were considered as significant.

Thomas Jefferson University Institutional Review Board approved this retrospective record review and database study. No informed consent was required, as it was a retrospective study.

\section{Results}

From 1986 until 2015, 1225 patients had epilepsy surgery; 978 patients had data on preoperative seizure clustering available; data was missing for 247 patients. Of the 978 patients included in this analysis, $681(69.6 \%)$ had temporal lobe surgery and $83(8.5 \%)$ had extratemporal surgery and were included in this analysis; we excluded 214 patients who had corpus callosotomy, vagus nerve stimulation, or multilobar resections with both temporal and extratemporal resection. The proportion of temporal resections among patients with missing data was similar to the studied group [of 247 patients with missing data, $161(65.2 \%)$ had temporal lobe surgery $(\mathrm{p}=0.1)$ ]. However, relatively more patients with missing data had extratemporal surgery [39 (15.8\%) patients; $\mathrm{p}=0.0001]$. Among patients with temporal lobe epilepsy (TLE), 161 (23.6\%) experienced seizure clusters and among extratemporal epilepsy patients, $14(16.9 \%)$ reported such an experience $(\mathrm{p}=0.2)$. Among patients with extratemporal surgery, having seizure clusters were not significantly different between patients with frontal lobe surgery (10 out of 60) compared with those with only parietal or occipital lobe surgery ( 4 out of 23$) ; p=1$. We could not identify any significant clinical associations for experiencing seizure clusters for the entire cohort (Table 1). Similarly, 
we could not identify any significant associations with clusters for TLE patients (all $\mathrm{p}$ values were above 0.06 ). In patients with TLE, neither side of surgery nor presence or absence of mesial temporal sclerosis (MTS) was associated with seizure clustering. Among patients with seizure clusters, 41 patients had normal MRI, 66 had MTS, and 52 had other lesions (2 MRIs were missing). Among patients with no seizure clusters, these figures were 105, 237, and 161, respectively (17 MRIs were missing) $(\mathrm{p}=0.3)$. There was no difference in the need for intracranial EEG recordings in patients with seizure clusters (21 out of 175) versus those without seizure clusters (87 out of 589) in the entire cohort $(p=0.3)$.

Next, we studied whether seizure clusters related to seizure outcome after focal resective epilepsy surgery. Among patients with TLE, those with a history of seizure clusters fared better after surgery than those patients who did not have clusters $(\mathrm{p}=0.005)$ (Fig 1). In patients with extratemporal epilepsy we did not observe such an association $(\mathrm{p}=0.09)$.

\section{Discussion}

Seizure clusters are relatively common phenomena among patients with epilepsy, particularly among those with drug-resistant seizures. About one fifth of our patients displayed this tendency. While we could not identify any distinct associations between seizures clusters and particular clinical or historical features, we observed that a history of seizure clusters was associated with a better seizure outcome after temporal lobe surgery. One of the most important risk factors associated with worse outcome after surgery for drug-resistant TLE is a history of preoperative tonic-clonic seizures ${ }^{5}$. In the current study, the prevalence of preoperative tonic-clonic seizures was similar in patients with and without seizure clusters, so clustering appears to be an independent prognostic factor. Hence, seizure clusters are a marker that the primary 
epileptogenic zone is more likely to be located in anterior and mesial temporal structures. More patients with extratemporal epilepsy need to be studied before firm conclusions can be drawn about the relationship between seizure clusters and postsurgical outcome.

Why seizures cluster is uncertain. We did not investigate the neurobiological underpinnings of seizure clusters in this study. However, previous studies suggest that it is possible that endogenous hormone fluctuations may lead to periods of enhanced seizure susceptibility and other periods with greater resistance to seizure recurrence ${ }^{6}$. Periods of enhanced susceptibility might then have more seizures, appearing as clusters. For example, the density of dendritic spines on hippocampal pyramidal cells is dependent on circulating estradiol and progesterone and fluctuates naturally during the estrous cycle in female rats. Changes in dendritic spine density during the estrous cycle are likely to have consequences for hippocampal neuronal excitability ${ }^{7}$. Other endogenous hormones could have similar effects. Hence, the cycling of seizures may correspond to these structural changes.

Because, seizure clustering may impose significant risks to patients, identifying the potential clinical associations is important. While we could not identify any associations or risk factors associated with seizure clustering, a previous study found that head trauma and extratemporal epilepsy were associated with seizure clusters ${ }^{3}$. Unfortunately, our database did not contain reliable descriptions of head trauma, so we could not assess this relationship. We could not replicate the previously reported association of extratemporal epilepsy with seizure clusters. In another study ${ }^{8}$, age at epilepsy onset was lower in cluster patients than in the non-cluster patients. We could not replicate this findings either. Given the lack of clear clinical associations, future efforts are probably better devoted to investigating other features that might shed light on the neurobiology of seizure clusters. 
How seizure clusters are defined ${ }^{9}$ might influence data collection, data interpretation, and study results, which is a potential limitation of this study. Any definition of seizure clusters is of necessity arbitrary and clusters probably exist along a spectrum with occurrence possibly influenced by the underlying neurobiology of seizures, medications, and other factors. We have used a broad definition to account for the clinical picture, and recognize that a stricter definition requiring seizures to occur over a period of a few hours might yield different results. Another limitation of this study is the reliability of the history of seizure clusters, as it was a retrospective study and recall bias is a potential limitation. Patients who were not seizure-free after surgery were not systematically re-assessed to explain the reasons for postsurgical failure. These reasons are likely to be different in people with temporal and extratemporal epilepsies, which could be a confounder of this study, but there were unlikely to be systematic differences in extent of resection or other features that would otherwise explain the difference. Furthermore, we did not take into account other factors that may impact postoperative outcome ${ }^{10-13}$. Finally, our findings may not apply to the overall group of patients with uncontrolled seizures, as we had a special subgroup of patients with drug-resistant focal epilepsy requiring surgery. Our findings apply to the patients who had anterior temporal lobectomy for drug-resistant temporal lobe epilepsy and not to other populations.

In brief, we found that seizure clusters relate to prognosis after anterior temporal lobectomy in drug-resistant TLE. This data may provide added value for surgical prognostication when combined with other data types $5,10-13$. A better understanding of the neurobiology underlying seizure clusters is needed. 


\section{Acknowledgment}

This study was not funded. We confirm that we have read the Journal's position on issues involved in ethical publication and affirm that this report is consistent with those guidelines.

\section{Contributions}

Ali A. Asadi-Pooya, M.D.: Study design, data collection, statistical analysis, manuscript preparation.

Maromi Nei, M.D.: Data collection, manuscript preparation.

Ashwini Sharan: Data collection, manuscript preparation.

Michael R. Sperling, M.D.: Study design, data collection, statistical analysis, manuscript preparation.

\section{Disclosures}

Ali A. Asadi-Pooya reports no conflicts of interest.

Maromi Nei, Research: Research: Upsher-Smith Laboratories, NINDS.

Ashwini Sharan: Consulting, Clinical Trial Site - Medtronic; SJM, Clinical Trial Site - Grant, honorarium; ICVRX - ownership interest; ICP - ownership interest; Tiger labs - ownership interest; Saluda Medical - Clinical Trial Site.

Michael R. Sperling: Research: Eisai, UCB Pharma, Sunovion, SK Life Sciences, Marinus, Lundbeck, Medtronics, Accorda, Upsher-Smith, Brain Sentinel, Glaxo, Pfizer. Dr. Sperling serves as the Editor-in-Chief of Epilepsia. 


\section{Reference}

1. Haut SR. Seizure clustering. Epilepsy Behav 2006; 8: 50-55.

2. Haut SR, Lipton RB, LeValley AJ, et al. Identifying seizure clusters in patients with epilepsy. Neurology 2005; 65: 1313-1315.

3. Haut SR, Shinnar S, Moshé SL. Seizure clustering: risks and outcomes. Epilepsia 2005; 46: $146-149$.

4. Sperling MR, O'Connor MJ, Saykin AJ, et al. Temporal lobectomy for refractory epilepsy. JAMA 1996; 276: 470-475.

5. Fong JS, Jehi L, Najm I, et al. Seizure outcome and its predictors after temporal lobe epilepsy surgery in patients with normal MRI. Epilepsia 2011; 52: 1393-1401.

6. Herzog AG. Catamenial epilepsy: definition, prevalence pathophysiology and treatment. Seizure; 17: 151-159.

7. Woolley CS, Gould E, Frankfurt M, et al. Naturally occurring fluctuation in dendritic spine density on adult hippocampal pyramidal neurons. J Neurosci 1990; 10: 4035-4039.

8. Bauer J, Burr W. Course of chronic focal epilepsy resistant to anticonvulsant treatment. Seizure 2001; 10: 239-246.

9. Haut SR. Seizure clusters: characteristics and treatment. Curr Opin Neurol 2015; 28: 143150.

10. Tonini C, Beghi E, Berg AT, et al. Predictors of epilepsy surgery outcome: a metaanalysis. Epilepsy Res 2004; 62: 75-87.

11. Spencer SS, Berg AT, Vickrey BG, et al; Multicenter Study of Epilepsy Surgery. Predicting long-term seizure outcome after resective epilepsy surgery: the multicenter study. Neurology 2005; 65: 912-918. 
12. Asadi-Pooya AA, Nei M, Sharan A, et al. Type of preoperative aura may predict postsurgical outcome in patients with temporal lobe epilepsy and mesial temporal sclerosis. Epilepsy Behav 2015; 50: 98-100.

13. Asadi-Pooya AA, Nei M, Sharan A, Sperling MR. Historical risk factors associated with seizure outcome after surgery for drug-resistant mesial temporal lobe epilepsy. World Neurosurg 2016; 89: 78-83. 
Figure 1. Kaplan-Meier graph showing cumulative time-dependent probability of occurrence of the first seizure after resective epilepsy surgery in patients with temporal lobe epilepsy, with or without preoperative seizure clusters.

Figure 1 legend. Postoperative seizure outcome was better in patients with drug-resistant temporal lobe epilepsy who had history of preoperative seizure clusters. 
Table 1. Clinical factors in association with seizure clusters

\begin{tabular}{|c|c|c|c|}
\hline Clinical characteristic & $\begin{array}{c}\text { Patients with } \\
\text { Seizure clusters } \\
\qquad(\mathrm{N}=175) *\end{array}$ & $\begin{array}{l}\text { Patients without } \\
\text { Seizure clusters } \\
\qquad(\mathrm{N}=589) *\end{array}$ & $\begin{array}{c}\mathbf{P} \\
\text { value }\end{array}$ \\
\hline Sex ratio (Male / Female) & $93 / 82$ & $284 / 305$ & 0.2 \\
\hline History of childhood febrile seizure & 46 & 148 & 0.8 \\
\hline Family history of epilepsy & 49 & 181 & 0.5 \\
\hline $\begin{array}{l}\text { Aura (none/ epigastric / auditory / } \\
\text { mnemonic / affective / others / multiple) }\end{array}$ & $\begin{array}{l}40 / 28 / 2 / 11 / 12 / \\
56 / 23\end{array}$ & $\begin{array}{l}197 / 85 / 17 / 37 / 24 \\
/ 167 / 58\end{array}$ & 0.08 \\
\hline $\begin{array}{l}\text { Remote history of tonic-clonic seizures } \\
\text { (not within the past year) }\end{array}$ & 48 & 159 & 0.9 \\
\hline $\begin{array}{l}\text { Recent history of tonic-clonic seizures } \\
\text { (within the past year) }\end{array}$ & 51 & 207 & 0.1 \\
\hline Age at epilepsy onset (years) & $15 \pm 11.1$ & $15.8 \pm 12.4$ & 0.4 \\
\hline Disease duration (years) & $18.8 \pm 11.5$ & $19.7 \pm 11.7$ & 0.3 \\
\hline Full scale IQ & $93 \pm 18$ & $92 \pm 15$ & 0.3 \\
\hline
\end{tabular}

*Some data were missing. 